\title{
Climatology and characteristics of stratospheric sudden warmings in the Whole Atmosphere Community Climate Model
}

\author{
Laura de la Torre, ${ }^{1,2}$ Rolando R. Garcia, ${ }^{2}$ David Barriopedro, ${ }^{3,4}$ and Amal Chandran ${ }^{2,5}$ \\ Received 7 September 2011; revised 19 December 2011; accepted 27 December 2011; published 25 February 2012.
}

[1] Major stratospheric sudden warmings (SSW) occurring during Northern Hemisphere winter were identified in four runs of the Whole Atmosphere Community Climate Model (WACCM). Their characteristics are compared to those found by other authors using reanalysis data. The comparison shows that the frequency of occurrence of major SSW in the model is very similar to that found in reanalysis data, as is the occurrence of vortex splitting and displacement events. The main difference with respect to observations is that the modeled SSW are relatively longer lasting. WACCM simulates quite accurately some dynamical features associated with major SSW, despite the presence of outlier cases; however, the recently reported relationship between regional blocking and the type of SSW is only partially reproduced by WACCM. In general, the observed climatological and dynamical signatures of displacement SSW tend to be better reproduced by the model than those associated with splitting SSW. We also find that SSW in the model are often associated with an elevated polar cap stratopause, in agreement with recent observations. However, the simulations also show that there is not in general a close correspondence between major SSW and elevated polar cap stratopause events.

Citation: de la Torre, L., R. R. Garcia, D. Barriopedro, and A. Chandran (2012), Climatology and characteristics of stratospheric sudden warmings in the Whole Atmosphere Community Climate Model, J. Geophys. Res., 117, D04110, doi:10.1029/2011JD016840.

\section{Introduction}

[2] During winter, the extratropical stratospheric circulation is characterized by westerly winds around a cold pole, the so-called stratospheric polar vortex. The vortex forms as the polar stratosphere cools after the autumn equinox; it becomes stronger in the middle of the winter; and it finally breaks down during spring with the establishment of the summer regime, which is characterized by easterly winds around a warm pole. During the life cycle of the vortex, planetary Rossby waves propagating upward from the troposphere disrupt its zonal symmetry and displace it from the pole. When the vortex is displaced from the pole, the polar temperature increases quickly and a sudden stratospheric warming (SSW) occurs. If, in addition, the zonal wind becomes easterly at $10 \mathrm{hPa}$ and $60^{\circ}$ of latitude, the SSW is known as a "major" warming. Major SSW are a common feature of the Northern Hemisphere stratosphere, but are almost absent in the observational record for the Southern \footnotetext{
Spain.

${ }^{1}$ EPhysLab, Facultad de Ciencias, Universidad de Vigo, Ourense,

${ }^{2}$ National Center for Atmospheric Research, Boulder, Colorado, USA.

${ }^{3}$ Instituto Dom Luiz, Universidade de Lisboa, Lisbon, Portugal.

${ }^{4}$ Departamento de Astrofísica y Ciencias de la Atmósfera, Instituto de Geociencias, Universidad Complutense de Madrid, Madrid, Spain.

${ }^{5}$ Geophysical Institute, University of Alaska Fairbanks, Fairbanks, Alaska, USA.
}

Copyright 2012 by the American Geophysical Union. 0148-0227/12/2011JD016840
Hemisphere, which contains but a single instance of a major SSW, in September 2002. Throughout the remainder of this paper, we use the abbreviation SSW to denote a major warming in the Northern Hemisphere, whether or not preceded by these qualifiers.

[3] Charlton and Polvani [2007] constructed a climatology of SSW using NCEP/NCAR and ERA-40 reanalyses. They found a frequency of occurrence of about six events per decade. In $46 \%$ of the cases the vortex splits into two pieces of roughly equal size (vortex splitting events), while in the remainder the vortex moves to lower latitude and an anticyclone takes its place over the pole (vortex displacement events). Charlton et al. [2007] compared the results found for the reanalysis with SSW characteristics computed from six stratosphere-resolving general circulation models (GCM). Their results showed that GCM are capable of simulating accurately the dynamics of SSW, but with lower frequency than observed. One of the models with poor results in the frequency of SSW was version 1 of the Whole Atmosphere Community Climate Model (WACCM1).

[4] Among tropospheric phenomena that have been related to the occurrence of SSW are blocking events [Martius et al., 2009; Woollings et al., 2010]. A blocking event occurs when a persistent anticyclone disrupts the tropospheric midlatitude westerlies. The associated perturbations in planetary wave activity may be part of the process that triggers the onset of the SSW. Martius et al. [2009] and Woollings et al. [2010] found that almost all the SSW found by Charlton and Polvani [2007] in the ERA-40 reanalysis 
are preceded by blocking patterns in the troposphere, and that the location of the blocking is related to the type of SSW. However, Taguchi [2008] tried to ascertain whether blocking events occur preferentially in association with SSW (before or after them) without finding a clear connection. Martius et al. [2009] and Woollings et al. [2010] argued that this apparent contradiction is partially due to the differences in the detection method for blocking events; Castanheira and Barriopedro [2010] further pointed out that, as the association is different depending on the type of SSW event, the grouping of all the blocking events together, as in the work of Taguchi [2008], could mask the relationship.

[5] Manney et al. [2008a] studied two strong SSW over Eureka $\left(80^{\circ} \mathrm{N}\right)$. In both cases, they observed that the vortex broke down throughout the stratosphere, reformed quickly in the upper stratosphere, and remained weak for a longer time in the middle and lower stratosphere. The polar stratopause dropped in altitude during the warming and then reformed at very high altitude, near $75 \mathrm{~km}$. Siskind et al. [2007] found that the very high stratopause in 2006 resulted from filtering by the disturbed stratospheric flow of gravity waves that would normally break near $50 \mathrm{~km}$ and above and that are critical in determining the climatological polar stratopause structure.

[6] The main objective of the present work is to compare the simulation of Northern Hemisphere SSW in a new version of WACCM with the wealth of observations that have become available over the last few decades, as described above. We show that WACCM reproduces well the behavior of major SSW in terms of frequency and dynamical characteristics. We also compare the relationship between tropospheric blocking and SSW in the model with that documented in recent studies. Finally, we analyze the behavior of the polar stratopause and the mean meridional circulation associated with warming events.

\section{Model, Data, and Methodology}

[7] We use the Whole Atmosphere Community Climate Model (WACCM), version 3.5.48, which is based upon the Community Atmosphere Model (CAM), developed at the National Center for Atmospheric Research (NCAR). WACCM encompasses the troposphere as well as the middle and upper atmosphere, up to about $140 \mathrm{~km}$ altitude and includes variable solar inputs (wavelength-resolved irradiance and energetic particle precipitation) [Garcia et al., 2007; Richter et al., 2010]. The present version also incorporates updates to the gravity wave parameterization, as well as a parameterization of turbulent mountain stress and relaxation of tropical winds to observations to produce a quasi-biennial oscillation (QBO), as described by Richter et al. [2010]. The horizontal resolution is $2.5^{\circ}$ longitude $\times$ $1.9^{\circ}$ latitude. The model has 66 vertical levels, and the vertical resolution varies from a little over $1 \mathrm{~km}$ in the lower stratosphere to about $3.5 \mathrm{~km}$ in the lower thermosphere.

[8] Four "reference" simulations, performed for the second Chemistry-Climate Model Validation (CCMVal-2) project of SPARC (Stratospheric Processes and their Role in Climate), are used to construct the model climatology of SSW in the Northern Hemisphere. In what follows, these four simulations are referred to as refb1.1 to refb1.4. They are independent realizations (each started from a slightly different initial condition) of the climate for the period 1953-2006, and use as boundary conditions observed sea surface temperatures as well as observed surface mixing ratios of $\mathrm{CH}_{4}, \mathrm{~N}_{2} \mathrm{O}, \mathrm{CO}_{2}$, and halogens. Here we analyze the period November 1954-March 2005, which yields 51 complete Northern Hemisphere winters (defined here as the months of November through March). Additional details on the boundary conditions can be found in the work of Eyring et al. [2010].

[9] We compare WACCM results against data from the NCEP/NCAR and ERA-40 reanalyses to validate the climatology of SSW in model, and we follow closely the methodology of Charlton and Polvani [2007] and Charlton et al. [2007] to characterize the SSW. In particular, we use Charlton and Polvani's [2007] detection criteria for SSW, to wit:

[10] 1. A major SSW occurs when the zonal-mean zonal wind at $60^{\circ} \mathrm{N}$ and $10 \mathrm{hPa}$ becomes easterly during Northern Hemisphere winter. This time is considered the central date of the SSW event.

[11] 2. No other SSW can begin within 20 days of a central date.

[12] 3. If the zonal-mean zonal wind does not return to westerly for at least 10 consecutive days before the end of the winter season, then the event is considered a final warming and excluded from the analysis.

[13] For each SSW, its central date, its amplitude and its duration were computed. The amplitude is defined as the mean area-weighted $\left(50^{\circ} \mathrm{N}-90^{\circ} \mathrm{N}\right)$ temperature anomaly (with respect to the climatological seasonal cycle) at $10 \mathrm{hPa}$ within \pm 5 days of the central date. The duration was computed as the number of consecutive days of easterly zonalmean zonal winds at $60^{\circ} \mathrm{N}$ and $10 \mathrm{hPa}$ after the central date. To distinguish between vortex splits and vortex displacements, a subjective analysis of the geopotential height field at $10 \mathrm{hPa}$ around the central date was used. If the geopotential field exhibited two maxima and two minima of approximately equal intensity, the SSW was classified as a splitting event; otherwise, the SSW was classified as a displacement event.

[14] The statistics used for the comparison with reanalysis data are described below. They were computed for the four runs as an ensemble (i.e., as a sample of $51 \times 4$ winters) to have as much data as possible and for each of the four runs separately to test the consistency of the results. The frequency of SSW occurrence per winter is addressed first, since Charlton et al. [2007] described it as the major shortcoming of numerical simulations of SSW. Other interesting parameters are the mean duration and amplitude of SSW, their monthly distribution, their interannual variability and the ratio between vortex split and vortex displacement events.

[15] To investigate the relationship between blocking events and SSW we follow the methodology of Martius et al. [2009]. First, a blocking detection method based on the work of Schwierz et al. [2004] was developed. We examine daily, de-seasonalized anomalies of the vertically averaged potential vorticity (between 150 and $500 \mathrm{hPa}$ ) with respect to the period of analysis and diagnose a blocking event when there is a closed contour of $-1.3 \mathrm{PVU}$ (the 10th percentile of the PV anomaly distribution) with an area of at least $1.8 \times$ $10^{6} \mathrm{~km}^{2}$ that persists for at least 5 days. A tracking algorithm is included, based on the percentage of spatial overlap 
Table 1. Total Number of Major SSW Events for the Periods Studied; Frequency of SSW per Winter; Ratio of Number of Displacement Versus Splitting SSW; Climatological Ratio of Area-Weighted, Winter-Mean Meridional Eddy Heat Flux (MHF) Between $45^{\circ} \mathrm{N}$ and $75^{\circ} \mathrm{N}$ at $100 \mathrm{hPa}$ Due to Wave Number 1 Versus Wave Number 2; and Mean Temperature Amplitude in the Polar Cap at $10 \mathrm{hPa}$ for Displacement and Splitting Events ${ }^{\mathrm{a}}$

\begin{tabular}{|c|c|c|c|c|c|c|}
\hline Case & Total SSW & SSW/Winter & Ratio Displacement/Split & MHF Ratio & Amplitude Displacement & Amplitude Split \\
\hline refb1.1 & 35 & $0.69(0.12)$ & 2.18 & 0.84 & $7.7(3.6)$ & $5.9(4.1)$ \\
\hline refb1.2 & 23 & $0.45(0.10)$ & 1.30 & 0.87 & $8.7(3.2)$ & $5.7(4.7)$ \\
\hline refb1.3 & 25 & $0.49(0.09)$ & 1.08 & 0.85 & $10.0(3.6)$ & $8.8(4.7)$ \\
\hline refb1.4 & 34 & $0.67(0.10)$ & 1.13 & 0.84 & $7.8(3.1)$ & $6.8(4.4)$ \\
\hline All WACCM & 117 & $0.57(0.10)$ & 1.39 & 0.85 & $8.4(3.4)$ & $6.9(4.5)$ \\
\hline NCEP & 27 & $0.60(0.10)$ & 1.25 & & $6.8(4.6)$ & $8.1(3.7)$ \\
\hline ERA & 29 & 0.64 & 1.10 & 0.66 & $7.4(4.8)$ & $9.1(4.9)$ \\
\hline NCEP+ERA & 28 & 0.62 & 1.18 & & $7.1(4.7)$ & $8.6(4.3)$ \\
\hline
\end{tabular}

${ }^{a}$ The numbers in parentheses are the standard errors, computed as in the work of Charlton et al. [2007]. The reanalysis figures are from Charlton and Polvani [2007] and Charlton et al. [2007]. The periods studied are 1954/55-2004/05 for WACCM, 1957/58-2001/02 for the reanalyses.

between successive daily blocks, which must exceed $45 \%$ for PV contours to be considered the same blocking event. This blocking detection algorithm has also been used in the work of Castanheira and Barriopedro [2010], where the reader may find details of its implementation. For the sake of simplicity, in the present analysis the original WACCM fields $\left(2.5^{\circ}\right.$ longitude $\times 1.9^{\circ}$ latitude $)$ were linearly interpolated to the ERA-40 $2.5^{\circ} \times 2.5^{\circ}$ grid, since the blocking detection method is insensitive to small changes in the spatial resolution. We use this method to obtain the blocking climatology for ERA-40 and WACCM and the frequency of occurrence of blocking for each grid point during 10 days before the onset of splitting and displacement warmings.

[16] In the third part of our analysis, the area-weighted mean polar cap temperature (northward of $70^{\circ}$ ) is used to study the polar stratopause behavior. We identify "elevated stratopause events" in terms of a rapid increase in the altitude of the polar cap stratopause, as follows:

[17] 1. Smooth the time series of polar cap temperature, $\mathrm{T}_{\mathrm{PC}}(z, t)$, with a 9 day running mean in time; this eliminates transient events wherein the altitude of the stratopause reverts quickly to its normal location and does not produce a persistent elevated stratopause;

[18] 2. Examine the smoothed time series of $\mathrm{T}_{\mathrm{PC}}$ to determine the position, $Z_{\mathrm{S}}$, of the polar stratopause, during Northern Hemisphere winter. The stratopause is defined as the altitude of maximum temperature in the interval $20 \mathrm{~km} \leq z \leq 100 \mathrm{~km}$;

[19] 3. Examine $Z_{\mathrm{S}}$ for day-over-day increases, and identify an elevated stratopause event at time, $t_{0}$, when there is a change $\Delta Z_{\mathrm{S}} \geq 15 \mathrm{~km}$. The time $t_{0}$ is the central date of the event.

[20] This procedure is applied in section 3.3 to WACCM simulation refb1.4. The composite evolution of all identified events and the behavior of a pair of specific events are discussed to highlight the general features of the phenomenon, its variability, and its relation to SSW. The behavior of elevated stratopause events in the model is compared with recent descriptions of the phenomenon by Manney and colleagues [Manney et al., 2008a, 2008b; Manney et al., 2009].

\section{Results}

\subsection{Characterization of SSW in WACCM}

[21] Table 1 shows that the frequency of major warmings per winter in WACCM (0.57) is similar to that found by Charlton and Polvani [2007] for the ERA-40 (0.64) and NCEP/NCAR (0.60) reanalyses. The differences among the individual runs are due to the internal variability of the model, as the four have the same boundary conditions. It is worth noting that the observed frequency of SSW can vary substantially depending on the period for which it is
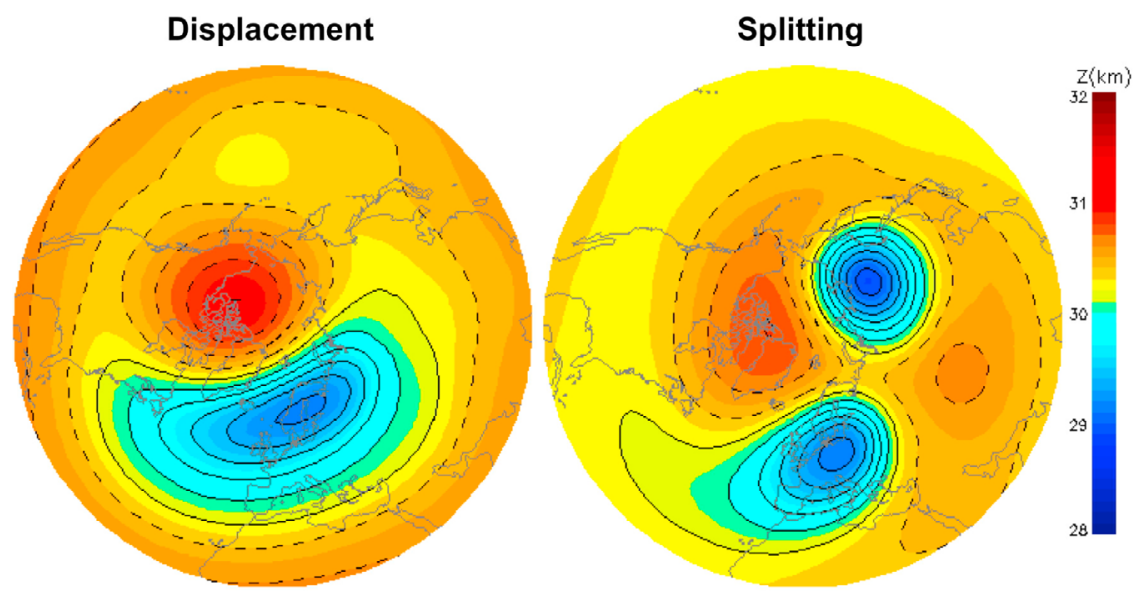

Figure 1. Geopotential height at $10 \mathrm{hPa}$ for examples of (left) displacement and (right) splitting major SSW events in WACCM. 
Table 2. Mean Duration of SSW Events, Computed as the Number of Consecutive Days of Easterly Zonal-Mean Zonal Winds at $60^{\circ} \mathrm{N}$ and $10 \mathrm{hPa}$ After the Onset of Each SSW, and Percentage of Short, Medium, and Long Events ${ }^{\mathrm{a}}$

\begin{tabular}{lcccc}
\hline & Mean Duration (days) & Percent $<$ 4 Days & Percent 4-9 Days & Percent $>9$ Days \\
\hline refb1.1 & 9.3 & 11.4 & 57.1 & 31.4 \\
refb1.2 & 9.6 & 13.0 & 47.8 & 39.1 \\
refb1.3 & 9.6 & 16.0 & 40.0 & 44.0 \\
refb1.4 & 8.3 & 17.6 & 50.0 & 32.3 \\
All WACCM & 9.1 & 14.5 & 49.3 & 35.9 \\
Displ. WACCM & 7.8 & 22.1 & 54.4 & 23.5 \\
Split WACCM & 10.9 & 4.1 & 42.9 & 53.1 \\
NCEP & 5.2 & 44.5 & 48.1 & 7.4 \\
\hline
\end{tabular}

${ }^{a}$ Rows 1 through 5 show WACCM results for all SSW in each simulation, and in the ensemble of all simulations, without distinguishing between displacement and splitting events. Rows 6 and 7 show WACCM ensemble results for displacement and splitting events separately. The NCEP/NCAR reanalysis figures for all SSW regardless of type (last row) are from Charlton et al. [2007]. Short events are less than 4 days; medium events are 4 to 9 days; and long events are more than 9 days.

computed. For example, using 30 year periods, the SSW frequency in the NCEP/NCAR reanalysis varies from 0.47 per winter (1960-1989) to 0.67 per winter (1972-2001).

[22] WACCM reproduces both vortex displacement and vortex splitting SSW (Figure 1). There is good agreement between the model and the reanalyses in the ratio of the two types of event, except for simulation refb1.1 (Table 1), where the ratio is almost double that in the other simulations. Charlton and Polvani [2007] found that this ratio (1.25 in NCEP/NCAR and 1.10 in ERA-40) can also vary substantially in observations: for example, over the period 1978/79 to $2001 / 02$, which is well documented by satellite observations, they found a ratio of 1.8 in the NCEP/NCAR reanalysis and 1.7 in the ERA-40 reanalysis. This variability is similar to what is found in the model, although the period of comparison (24 winters) is much shorter than the 51 winters in the WACCM simulations. Thus, the difference between simulation refb1.1 and observations appears less dramatic when considered in the context of observed variability.

[23] To further explore the differences in the relative frequency of SSW, the climatological ratio of the $45^{\circ} \mathrm{N}$ to $75^{\circ} \mathrm{N}$ area-weighted winter-mean meridional eddy heat flux (MHF; $\overline{v^{\prime} T^{\prime}}$ ) at $100 \mathrm{hPa}$ due to wave number 1 and wave number 2 was also computed. The higher meridional eddy heat flux ratio in WACCM (Table 1) indicates that wave

a) monthly frequency SSW-all events

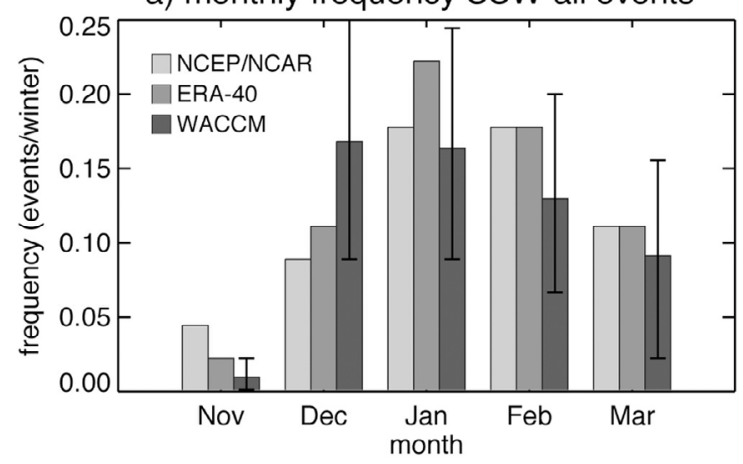

b) monthly frequency SSW-displacements

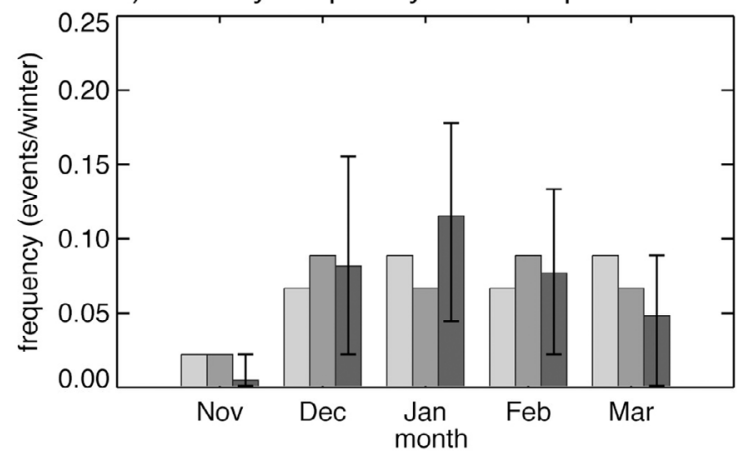

c) monthly frequency SSW-splits

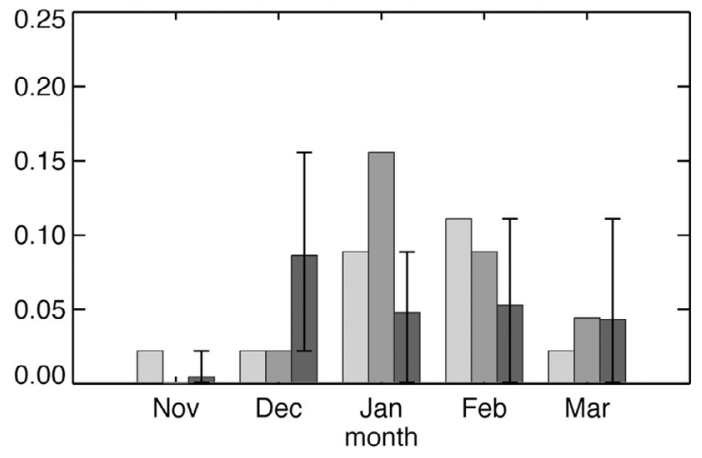

Figure 2. Monthly frequency of major SSW events for WACCM, NCEP/NCAR reanalysis and ERA-40 reanalysis. (a) All SSW events and (b and c) SSW shown separately for displacement and splitting events. The reanalysis statistics are from Charlton and Polvani [2007]. The WACCM results show the 5th and 95th percentile limits from a Monte Carlo test. See text for details. 
monthly amplitude of all events

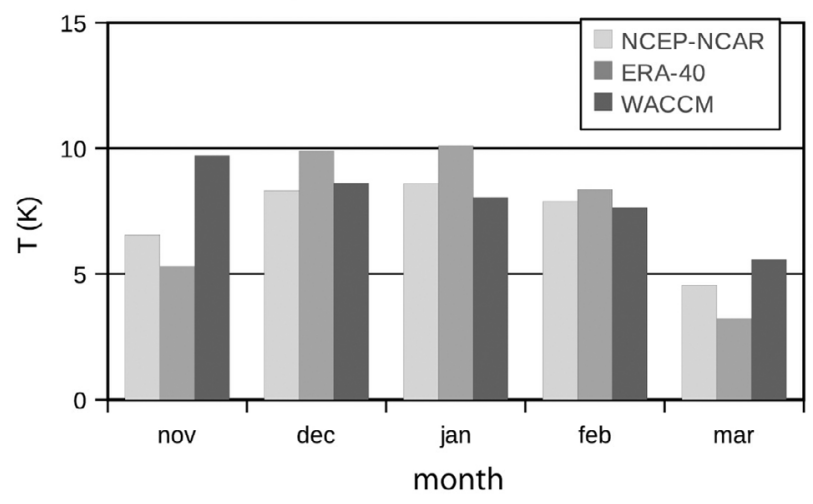

interannual amplitude of SSWs

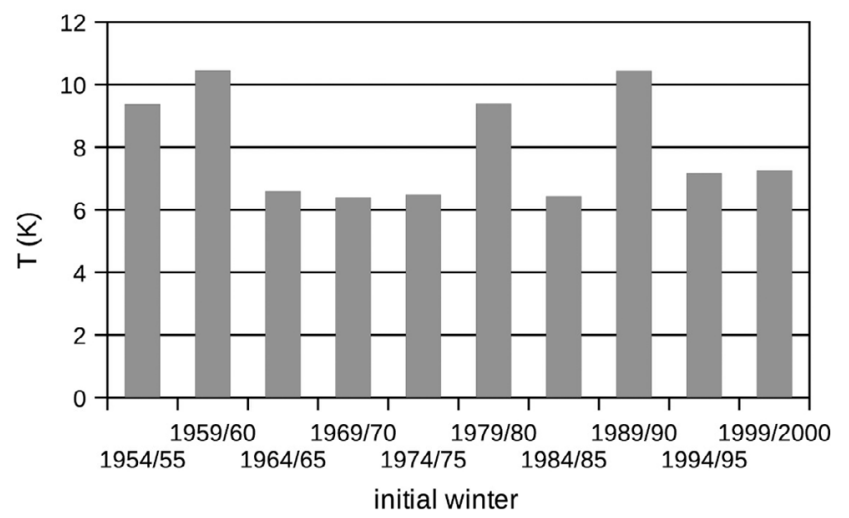

Figure 3. (left) Monthly and (right) interannual distributions of the temperature amplitude of WACCM SSW events. The monthly distribution compares WACCM results against NCEP/NCAR and ERA-40 data. The interannual distribution shows the mean amplitude of warmings for pentads (5 year periods, beginning with the winter indicated on the abscissa).

number 1 planetary wave is more important than wave number 2 in the model. In the model, vortex splitting events usually involve a decrease in wave number 1 amplitude more than an increase in wave number 2 amplitude (not shown). In fact, the amplitude of wave number 2 is uniformly weak in WACCM simulations compared to observations. Along the same lines, the temperature amplitude in WACCM, unlike in the reanalysis, is larger for vortex displacement than for vortex splitting events. However, a Student's $t$ test of the results shown in Table 1 indicates that the difference between the amplitude of displacement and splitting events is not statistically significant at the $95 \%$ level for any of the individual simulations, and is barely significant at that level for the ensemble of all simulations.

[24] Table 2 shows that the mean duration of the events is $75 \%$ longer in WACCM than in NCEP/NCAR. The overall higher persistence of SSW in WACCM arises from a deficit of short-duration SSW ( $<4$ days) together with a surplus of long-duration SSW ( $>9$ days); the number of SSW of intermediate duration (4-9 days) is similar in WACCM and the reanalysis. Table 2 also shows that the event duration statistics for vortex displacement events in WACCM are much more similar to the reanalysis than the results for splitting events. Thus, most of the discrepancy in event duration between WACCM and the reanalysis arises from the tendency of splitting events to be very long lived in the model.

[25] Figure 2 shows monthly frequency statistics in WACCM compared to observations. For the WACCM frequencies, we also show an estimate of variability obtained from a Monte Carlo test. This test is performed by constructing a 1000 member ensemble of winters (NovemberMarch) from the output of the model. Each member of the ensemble consists of 45 winters (the same number as in the ERA-40 and NCEP/NCAR data sets), which are chosen randomly and without replacement from all the years in the four WACCM simulations. The monthly frequency of SSW is calculated for each ensemble member and, from this, the two-sided 5\% limits of the Monte Carlo distribution of monthly SSW frequencies are computed. These limits are indicated in Figure 2 by the range bars superimposed on the
WACCM SSW frequencies. The monthly frequency of all SSW in the model (Figure 2a) is comparable to the observed frequency during January through March; it is considerably higher during December and lower in November, but only in November is the difference statistically significant, and then only when compared to NCEP/NCAR data. If displacement and splitting events (Figures $2 \mathrm{~b}$ and $2 \mathrm{c}$ ) are considered separately, the frequency of the former is comparable to that found in the reanalyses in all months; therefore, the difference in overall SSW frequency is associated mainly with splitting events. The latter are significantly different from both reanalyses in November and December.

[26] The monthly temperature amplitude at $10 \mathrm{hPa}$ during major SSW in WACCM is shown in Figure 3 (left), together with the values obtained from the NCEP/NCAR and ERA40 reanalyses by Charlton and Polvani [2007]. The amplitude in November is not statistically reliable due to the small number of cases. Figure 3 (right) shows the amplitude of SSW in the model by pentad; the variability is similar to that reported for reanalysis data by Charlton and Polvani [2007, Figure $3 \mathrm{~d}]$.

[27] The distribution of SSW frequency by pentad (Figure 4) shows that the interannual variability in WACCM is similar to that found in the NCEP/NCAR and ERA-40 reanalyses by Charlton and Polvani [2007, Figures 3a-3c]. However, there are fewer pentads without SSW in the model than found in the reanalyses. There is an increase in the number of SSW until the 1970s, but afterward the number is comparable to that found in the 1950s and remains roughly constant. The variability is higher for vortex splitting events, which become less frequent after 1985. Figure 4 also shows that, for some periods, the interannual variability in SSW frequency is similar in all four WACCM simulations, while for other periods it is completely different across the simulations. The differences are presumed to be due to the internal variability of the model. As for the similarities, they might arise from the influence of factors such as ENSO and the QBO, which have been shown by Richter et al. [2011] to affect the overall frequency of occurrence of SSW in WACCM, although any such effect is far from obvious when considering interdecadal variability. The QBO in 

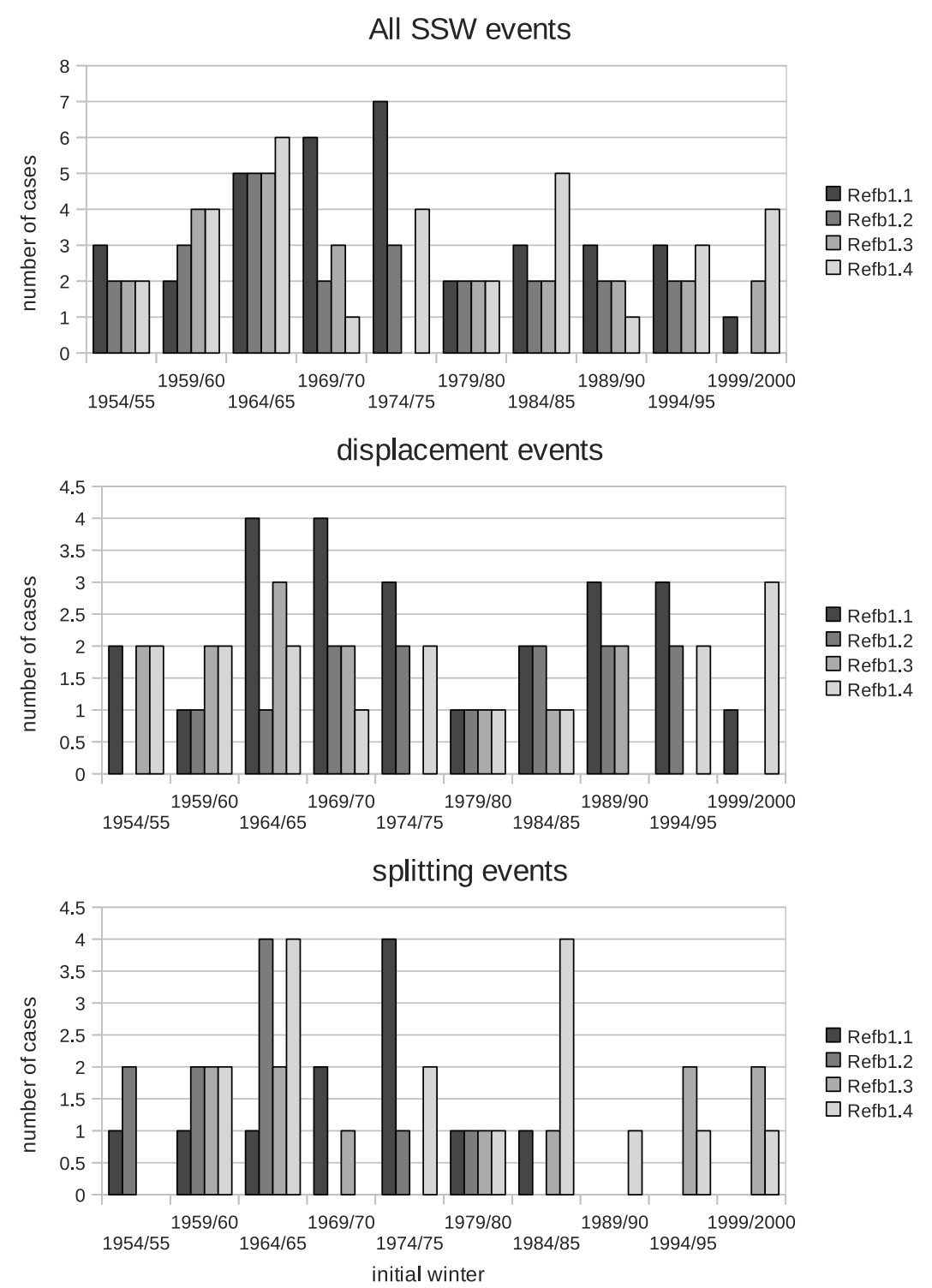

Figure 4. Distribution of SSW by pentad (5 year periods, beginning with the winter indicated on the abscissa) for all SSW, displacement events, and splitting events for each of the four WACCM simulations.

WACCM is forced by relaxation to observations of tropical winds and these observations do not indicate any remarkable changes in the behavior of the QBO since the 1950s. Similarly, while the intensity of ENSO events (as measured, for example, by a time series of the multivariate ENSO index [Wolter and Timlin, 2011]) exhibits interdecadal variability, there is no clear correspondence between such variability and those periods when the frequency of SSW is similar across all WACCM simulations. Thus, confirmation of any relationship between the QBO, ENSO, and the frequency of SSW during specific periods in the WACCM simulations requires additional, detailed analysis that is beyond the scope of the present study.

[28] Figure 5 shows box plots of four dynamical "benchmarks" of SSW, as defined by Charlton and Polvani [2007]: The polar cap temperature amplitude in the middle stratosphere; the polar cap temperature amplitude in the lower stratosphere; the deceleration of the polar vortex at $10 \mathrm{hPa}$ and $60^{\circ} \mathrm{N}$; and the mean meridional eddy heat flux anomaly at $100 \mathrm{hPa}$. All anomalies are defined with respect to the climatological seasonal cycle. The boxes show the interquartile range of the distribution of each benchmark. The bars and whiskers denote the extremes of the distribution that are not considered outliers. Outliers are defined as any values that lie more than 1.5 times the interquartile range from the ends of each box.

[29] The first benchmark in Figure 5 gives an indication of the amplitude of the SSW in the middle stratosphere; the second is a measure of the strength of downward extent of the temperature anomalies; the third indicates the rate of momentum deposition that accompanies the SSW; and, finally, the fourth benchmark is proportional to the vertical flux of planetary wave activity that drives the SSW. The mean values for these statistics in WACCM are $7.7 \mathrm{~K}, 3.0 \mathrm{~K}$, $20.6 \mathrm{~m} \mathrm{~s}^{-1}$, and $9.6 \mathrm{~K} \mathrm{~m} \mathrm{~s}^{-1}$. The corresponding means for the NCEP/NCAR reanalysis are $7.4 \mathrm{~K}, 2.0 \mathrm{~K}, 26.2 \mathrm{~m} \mathrm{~s}^{-1}$ and $8.5 \mathrm{~K} \mathrm{~m} \mathrm{~s}^{-1}$ [Charlton et al., 2007]. Whereas the representation of the polar temperature amplitude in the middle 


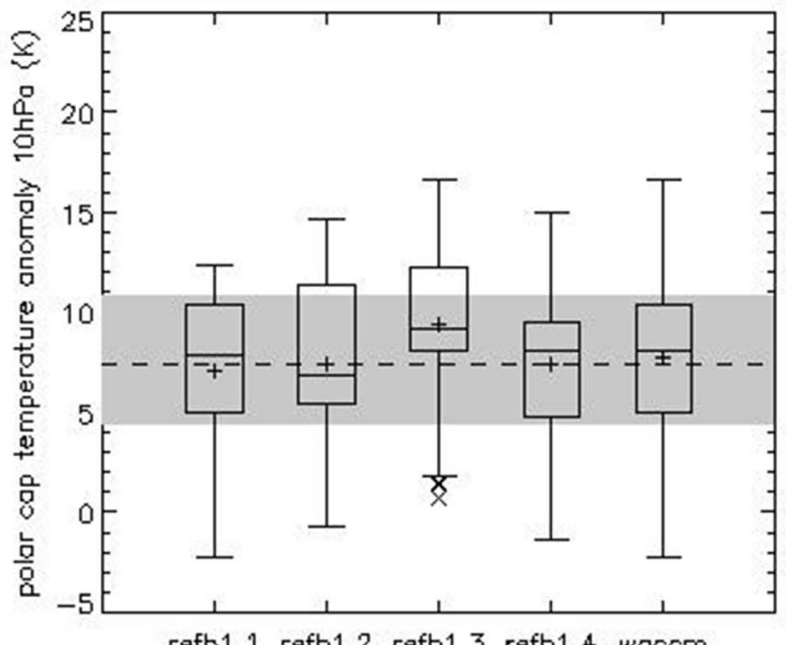

refb1.1 refb1.2 refb1.3 refb1.4 waccm

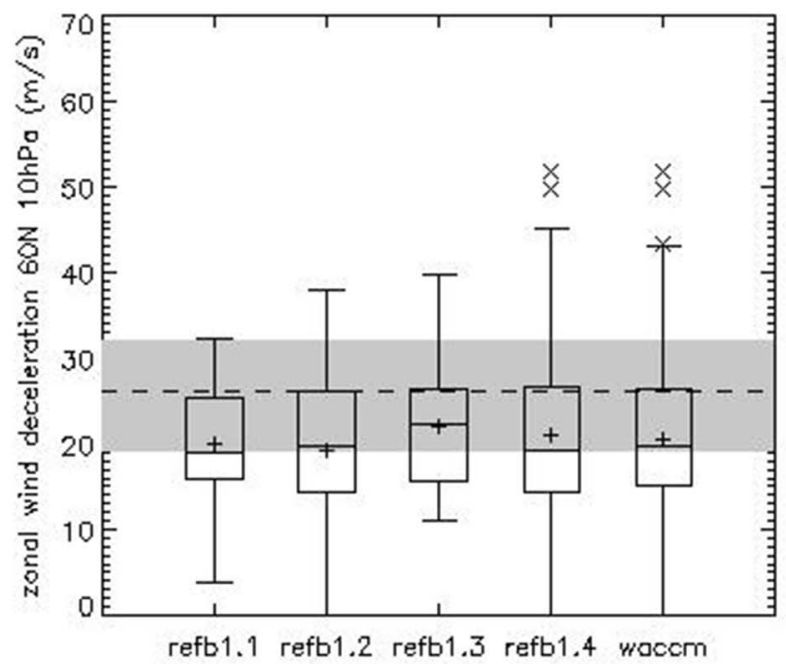

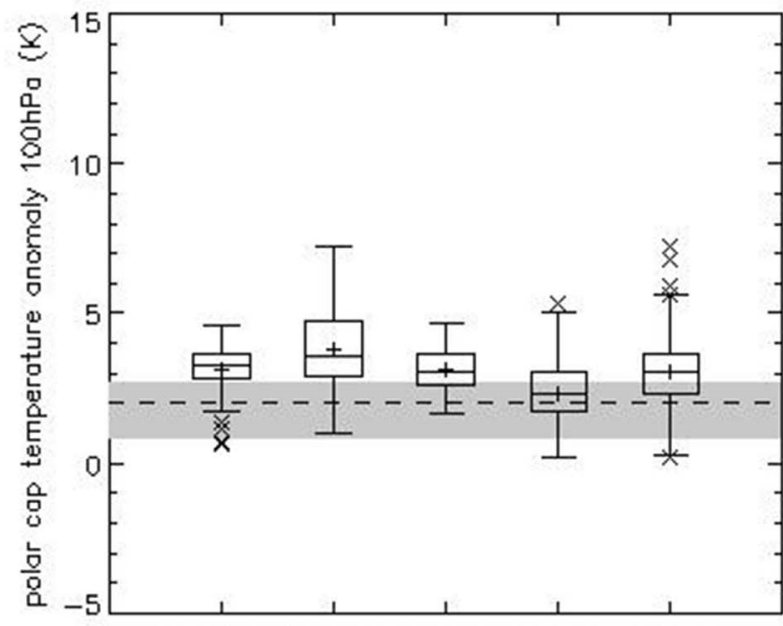

refb1.1 refb1.2 refb1.3 refb1.4 woccm

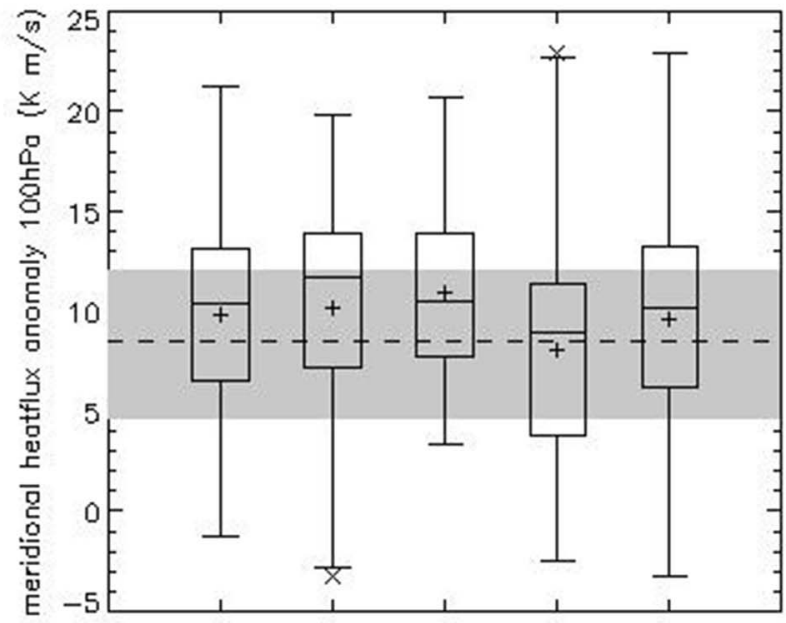

refb1.1 refb1.2 refb1.3 refb1.4 woccm

Figure 5. Dynamical benchmarks of major SSW in WACCM: (top) Polar cap temperature anomaly at $10 \mathrm{hPa}$ and $100 \mathrm{hPa}$, respectively, averaged over $50^{\circ}-90^{\circ} \mathrm{N}$ and \pm 5 days from the central date of the SSW; (bottom left) difference in the zonal-mean zonal wind at $10 \mathrm{hPa}$ and $60^{\circ} \mathrm{N}$ averaged over days $15-$ 5 minus days $0-5$ before the central date of the SSW; and (bottom right) meridional eddy heat flux anomaly at $100 \mathrm{hPa}$, averaged over $45^{\circ}-75^{\circ} \mathrm{N}$ and 20 to 0 days prior to the central date of the SSW. The size of each box indicates the interquartile range; the central line of the box shows the median; the mean value of the diagnostic is denoted by a cross, and the bars associated with each box denote the minimum and maximum points in the distribution that are not considered outliers. Outliers are marked by an "x." For comparison, the means and interquartile ranges for the same quantities in the NCEP/NCAR reanalysis [Charlton et al., 2007] are denoted, by the dashed line and shaded band in each plot, respectively.

stratosphere and the meridional eddy heat flux anomaly are very accurate in WACCM compared to observations, the amplitude in the lower stratosphere is somewhat too high and the polar vortex deceleration is slightly low. The extremes are, in general, more extreme in the model and in several cases there are outliers, whereas Charlton et al. [2007] found none in the reanalyses. Nonetheless, the SSW dynamical characteristics are well represented in WACCM overall.

[30] In the reanalyses, there is a fairly compact linear relationship between SSW amplitude in the middle stratosphere and the eddy heat flux anomaly at $100 \mathrm{hPa}$. This is also found in the model, as shown in Figure 6, where the correlation coefficient between the two benchmarks is 0.63 , the regression coefficient is 0.72 , and the coefficient of determination, $\mathrm{R}^{2}$, is 0.84 . These values are very similar to those found by Charlton et al. [2007] for NCEP/NCAR $(0.59,0.77$ and 0.86 , respectively).

\subsection{Relationship to Blocking Events}

[31] In this section, we first examine the ability of WACCM to capture the climatological features of blocking observed in the ERA-40 reanalysis using the algorithm described in section 2 . The association between blocking 


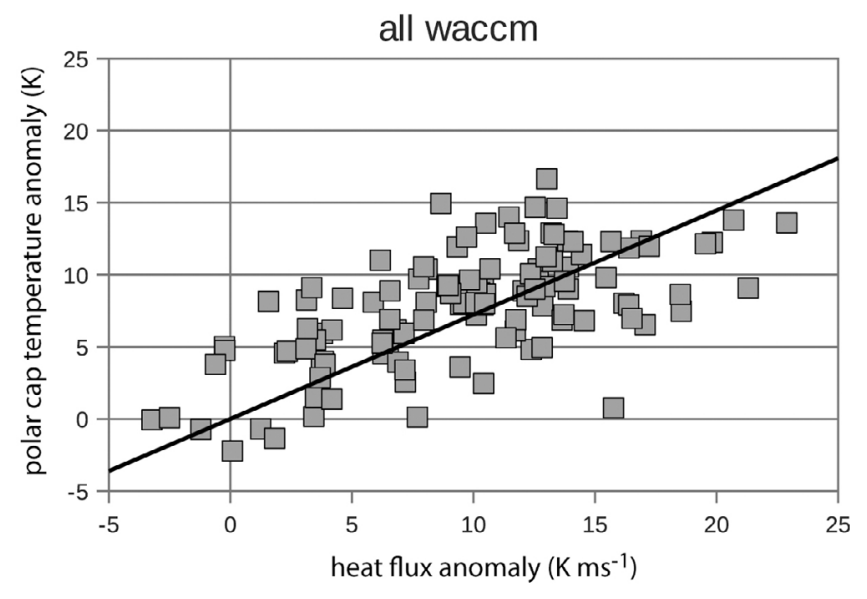

Figure 6. Scatterplot showing polar cap temperature anomaly averaged over $50^{\circ}-90^{\circ} \mathrm{N}$ and \pm 5 days from the central date of the SSW versus the meridional eddy heat flux anomaly at $100 \mathrm{hPa}$, averaged over $45-75^{\circ} \mathrm{N}$ and 20 to 0 days prior to the central date of the event, for all major SSW in WACCM. The black line is a linear least squares fit, calculated as in the work of Charlton et al. [2007]. See text for details.

and SSW in ERA-40 is then described, using the methodology already employed in previous studies. Finally, we explore the performance of WACCM in reproducing the observed linkages between regional blocks and the type of SSW.

[32] Figure 7 illustrates the mean blocking frequency in percentage of days during Northern Hemisphere winter for ERA-40 (1958-2001) and WACCM $(4 \times 51$ years $)$. Also shown in Figure 7 is the position of the upper tropospheric jet at $250 \mathrm{hPa}$, defined as the time-mean latitude of maximum zonal wind at each longitude, and indicated by the thick black lines in Figures 7 (top) and 7 (middle). WACCM reproduces the main observed signatures of blocking, namely, its preferred locations and the relative frequency between Atlantic and Pacific blocking. The difference in blocking frequency between WACCM and ERA-40 is shown in Figure 7c. Overall, there is a statistically significant tendency for WACCM to overestimate blocking occurrence, especially at latitudes south of the observed blocking maximum in the Pacific sector. Over the Atlantic sector, blocking activity in the model is in closer agreement with the reanalysis, except that the eastern edge of the center of action tends to be shifted eastward and extends further southward over Europe. This is consistent with a jet stream axis that is tilted NW-SE in the model relative to the reanalysis, as seen in Figure 7 [cf. Barriopedro et al., 2010]. On the other hand, in the Pacific sector, the position of the jet in WACCM agrees rather well with the reanalysis, although its speed is somewhat higher than observed (not shown). This suggests that the position of the jet cannot account for differences in blocking between WACCM and observations in the Pacific sector. The model bias in Pacific blocking frequency may be due instead to anomalously persistent subtropical ridging, since subtropical ridges tend to last longer than extratropical blocks and can cause slight northward shifts of the jet without reducing its speed [see, e.g., Woollings et al., 2011]. Indeed, we have ascertained that the blocks that account for most of the model's blocking bias in the Pacific sector relative to observations occur systematically at lower latitudes than blocks that do not contribute to the discrepancies between the model and the reanalysis. This suggests that the model does tend to overestimate the frequency of subtropical ridges. Incidentally, we find similar behavior for Euro-Atlantic blocks, but in this case it is
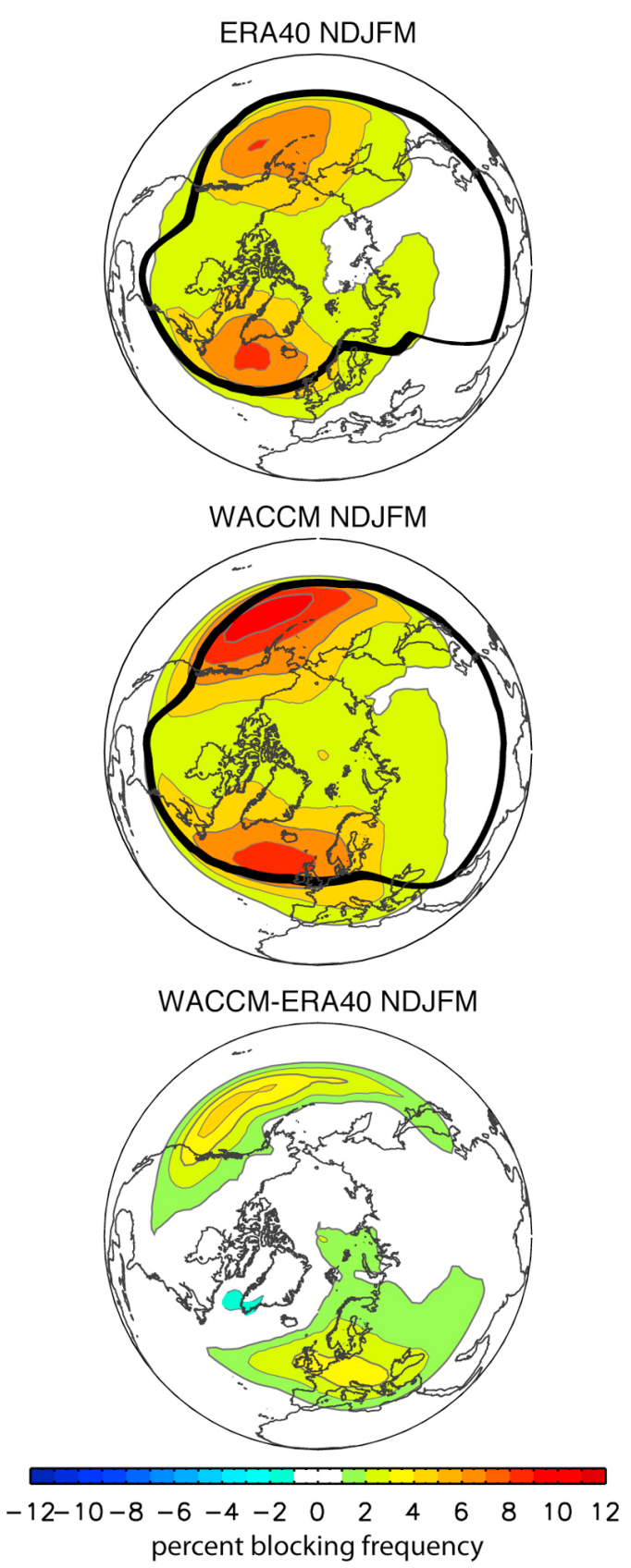

Figure 7. Mean blocking frequency in percentage of days during Northern Hemisphere winter for (top) ERA-40 (1958-2001) and (middle) WACCM. The thick black lines in the top and middle plots denote the mean position of the upper tropospheric jet at $250 \mathrm{hPa}$. (bottom) The difference between WACCM and ERA-40 blocking frequency is shown, where only differences significant at $\mathrm{p}<0.05$ according to a two-sided Student's $t$ test are depicted. See text for details. 
a) ERA40 before SSW-d

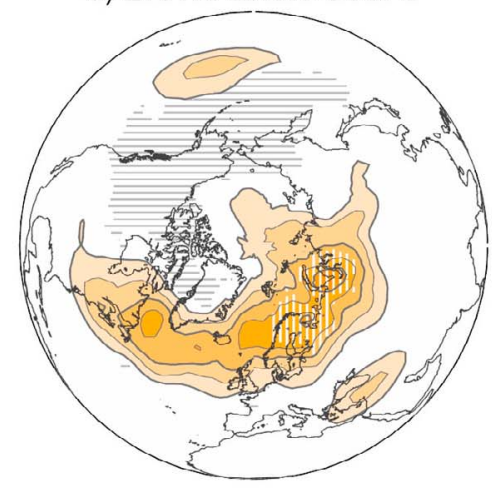

c) ERA40 before SSW-s

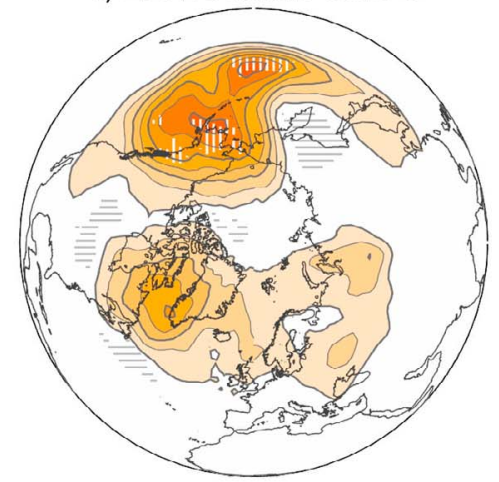

e) ERA40 before SSW s-d

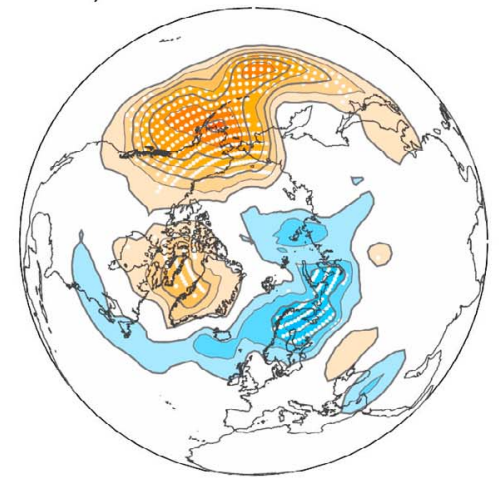

b) WACCM before SSW-d

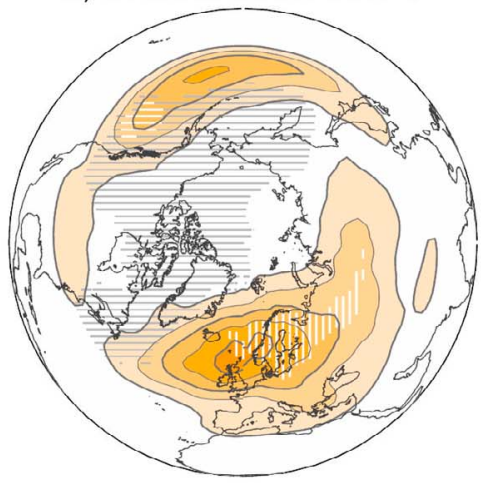

d) WACCM before SSW-s

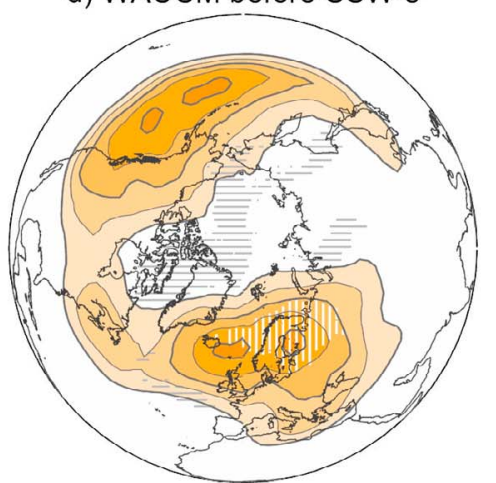

f) WACCM before SSW s-d

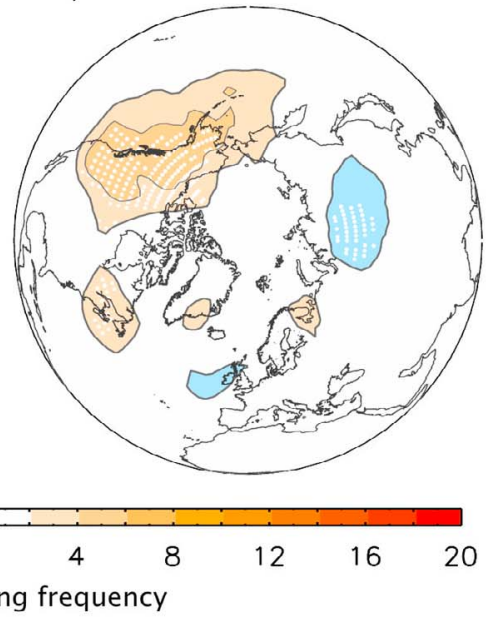

Figure 8. Composites of blocking frequency for the 10 day periods before the central date of (a and $b)$ displacement and (c and d) splitting SSW for ERA-40 reanalysis and WACCM; positive and negative significant differences with respect to climatology at the $95 \%$ confidence level are cross-hatched with vertical white lines and horizontal black lines, respectively. (e and f) The difference between splitting minus displacement SSW composites of blocking frequency for ERA-40 and WACCM, with white crosshatching indicating significant differences at $\mathrm{p}<0.05$. The significance was assessed with a Monte Carlo test (see text for details).

difficult to determine whether the blocking bias is due mainly to excessive subtropical ridging or to the bias in the mean location of the Euro-Atlantic jet discussed above.

[33] In spite of the shortcomings mentioned, Figure 7 shows that WACCM reproduces blocking activity reasonably well. Therefore, we have also compared the relationship between blocking and SSW in the model and in ERA-40.
Following the methodology employed by Martius et al. [2009], composites of blocking frequency for the 10 day period before the central date of displacement and splitting SSW have been computed for the ERA-40 reanalysis. In order to assess differences between blocking events associated with SSW and the climatology of all blocking events, a Monte Carlo test was performed by constructing, 
Table 3. Anomalies in the Number of Days With Blocking in the Euro-Atlantic and Pacific Sectors for the 20 Day Periods Before and After the Central Date of Major SSW

\begin{tabular}{lccc}
\hline Sector & Before & After & Before Minus After \\
\hline \multirow{3}{*}{ Atlantic } & $0.9 / \mathbf{1 . 2}$ & Displacement $S S W$ & \\
Pacific & $-\mathbf{- 1 . 8} /-\mathbf{- 3 . 3}$ & $-\mathbf{1 . 9} /-0.1$ & $\mathbf{2 . 7 / 3 . 0}$ \\
& & Splitting $S S W$ & $-1.3 /-\mathbf{3 . 2}$ \\
Atlantic & $-0.1 / 0.2$ & $-\mathbf{2 . 2} /-\mathbf{2 . 8}$ & $\mathbf{2 . 1 / 3 . 0}$ \\
Pacific & $\mathbf{2 . 4} /-\mathbf{1 . 2}$ & $1.5 /-0.4$ & $0.9 /-0.8$ \\
& & All $S S W$ & $\mathbf{2 . 3 / 2 . 9}$ \\
Atlantic & $0.3 / \mathbf{0 . 7}$ & $-\mathbf{2 . 0} /-\mathbf{2 . 2}$ & $-0.2 /-\mathbf{2 . 2}$ \\
Pacific & $-0.3 /-\mathbf{2 . 4}$ & $-0.1 /-0.2$ & \\
\hline
\end{tabular}

${ }^{\mathrm{a}}$ The fourth column shows the difference between composites before and after SSW. Numbers in each cell denote ERA-40/WACCM results. The anomalies are defined with reference to the climatological frequency, as derived from a Monte Carlo distribution of comparable 20 day periods. Bold type highlights significant departures from expectation at $\mathrm{p}<0.05$, and bold-italic type at $\mathrm{p}<0.1$ (see text for details). The Euro-Atlantic sector is $40^{\circ} \mathrm{W}, 50^{\circ} \mathrm{E}$, and the Pacific sector is $140^{\circ} \mathrm{E}, 230^{\circ} \mathrm{E}$.

for WACCM and ERA-40 each, a 1000 member "climatological ensemble." Each member of the ensemble contains as many 10 day periods as there are SSW events; these 10 day periods are chosen to coincide with the days and months when the SSW actually occur, but the year of occurrence is chosen at random. Thus, each Monte Carlo ensemble yields a climatological probability distribution of blocking during 10 day periods that occur at the same point of the seasonal cycle as the 10 day periods preceding SSW events. Then, for each grid point in latitude and longitude, significance at the 5\% level is attained when the 10 day blocking frequency before SSW is above the 95th or below the 5 th percentile of the probability distribution derived from the Monte Carlo ensemble.

[34] Figures 8a and 8c show the 10 day mean blocking frequency before displacement and splitting SSW, respectively, in the ERA-40 reanalysis. Cross-hatching is used to identify those regions where blocking activity is significantly different from the Monte Carlo climatology. These results are in good agreement with those reported by Martius et al. [2009]. These authors showed that, in ERA-40 data, Atlantic blocks tend to occur before displacement SSW while Pacific (or simultaneous Pacific and Atlantic) blocks precede splitting SSW. Figure 8a confirms that displacement SSW are characterized by an increase of Euro-Atlantic blocking and a suppression of Pacific blocking with respect to climatology. On the other hand, blocking frequency before splitting SSW (Figure 8c) is only significantly different from climatology in the Pacific basin, where a remarkable blocking increase with respect to the climatological mean occurs. The difference in blocking frequency between splitting and displacement SSW (Figure 8e) indicates that blocking activity is enhanced in the Pacific sector and reduced in the Euro-Atlantic sector before splitting SSW as compared with displacement SSW. However, in the EuroAtlantic sector significant differences between the two types of event are confined mostly to northern Europe. This suggests that Atlantic blocking may play a role in triggering both types of SSW [Martius et al., 2009; Woollings et al.,
2010]. Castanheira and Barriopedro [2010] further emphasized that the nearly contemporaneous occurrence (i.e., within 10 days) of Pacific and Atlantic blocking reported by Martius et al. [2009] before splitting SSW could in fact hide a small time delay, since the amplification of wave number 2 prior to splitting SSW is in turn preceded by amplification of wave number 1, typically associated with Euro-Atlantic blocks.

[35] A similar analysis to that of Castanheira and Barriopedro [2010] has been performed by computing the frequency of days when a blocking pattern was detected over the Euro-Atlantic $\left(40^{\circ} \mathrm{W}, 50^{\circ} \mathrm{E}\right)$ and Pacific $\left(140^{\circ} \mathrm{E}\right.$, $230^{\circ} \mathrm{E}$ ) sectors during the 20 days before the central date of each SSW. Regional blocking sectors and a wider temporal window were chosen to increase sample size and to account for the possible lag between Atlantic and Pacific blocks in SSW of the splitting type. Results from the ERA-40 reanalysis, shown in Table 3, support most of the aforementioned characteristics, in particular the increase of Pacific blocking with respect to climatology before splitting SSW and its decrease before displacement events. This analysis also confirms that Euro-Atlantic blocking activity is significantly reduced following SSW of any type, which is in agreement with a reported preconditioning of the polar vortex, according to which a weakening of the polar vortex tends to suppress Euro-Atlantic blocking [Castanheira and Barriopedro, 2010; Woollings et al., 2010].

[36] The same methodology used to analyze ERA-40 data is applied next to the SSW in the $4 \times 51$ years of WACCM output; the results are shown in Figure 8 (right). The model is able to simulate some of the reported linkages between blocking and SSW, particularly those for the displacement type. Thus, displacement SSW in the model are also preceded by statistically significant reduction of Pacific blocking and an increase of Euro-Atlantic blocking with respect to climatology (Figure 8b). However, the almost total absence of Pacific blocking before displacement SSW observed in ERA-40 (Figure 8a) is not seen in WACCM. This may be due to mean biases in the model, which shows a tendency to over represent Pacific blocking at midlatitudes (Figure 7c). As regards splitting SSW (Figure 8d), WACCM exhibits a greater frequency of Pacific blocks before splitting events than before displacement events, in agreement with ERA-40 (Figure 8c). However, the frequency of Pacific blocks is not significantly higher than climatology in the model, whereas it is so in ERA-40. Furthermore, WACCM shows a significant increase in Euro-Atlantic blocking with respect to climatology before splitting SSW that is not seen in ERA-40. Due to the model's failure to reproduce accurately the observed regional connections between the types of SSW and the patterns of blocking frequency, the simulated difference in blocking patterns before splitting and displacement SSW (Figure 8f) shows reduced amplitude and significance compared to ERA-40 (Figure 8e).

[37] An analysis of the frequency of blocking days in the Euro-Atlantic and Pacific sectors was also performed for WACCM; the results are shown in Table 3 next to those from ERA-40. The observed behavior of Euro-Atlantic blocking is relatively well reproduced by the model for both types of SSW; in particular, in agreement with ERA-40, WACCM simulates a significant reduction in Euro-Atlantic blocking following both types of SSW. In the case of Pacific 


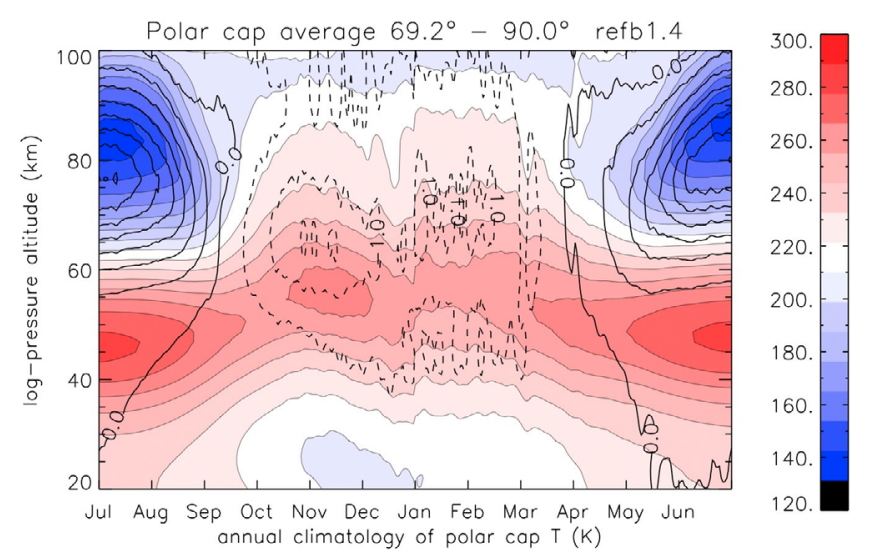

Figure 9. Climatology of WACCM polar cap temperature (K; filled contours, color scale on right) and vertical component of the TEM circulation, $\bar{w}^{*}\left(\mathrm{~cm} \mathrm{~s}^{-1}\right.$; line contours at $0.5 \mathrm{~cm} \mathrm{~s}^{-1}$ intervals, with negative values denoted by dashed contours), for simulation refb1.4. The vertical coordinate in this and all subsequent figures is $\log$ pressure altitude, $z_{\mathrm{P}}=$ $H \ln (1000 / p)$, where $p$ is pressure and $H=7 \mathrm{~km}$.

blocking, there are important discrepancies between WACCM and ERA-40, which arise principally from the model's inability to reproduce the observed enhancement of blocking frequency with respect to climatology before splitting SSW; in fact, WACCM produces a statistically significant decrease in this case.

[38] It is tempting to attribute some of the discrepancies between WACCM and observations seen in Figure 8 to the systematic errors in the model's blocking climatology discussed earlier (Figure 7). However, such attribution is not always straightforward. For example, some regions where the observed relationship between SSW and blocking is not well captured by the model, such as western Greenland (compare Figures 8e and 8f), do not display systematic model biases in blocking frequency (Figure 7c). On the other hand, regions that do have significant model biases in blocking frequency do not show consistent biases in the relationship between SSW and blocking. Thus, the model's blocking frequency in the Pacific sector before displacement events is higher than in ERA-40 (Figures 8c and 8d), as is the overall bias in blocking frequency in that sector (Figure 7c); on the other hand, the model's blocking frequency in the Pacific sector before splitting SSW is weaker than observed. Therefore, it is not clear to what extent deficiencies in the relationship between blocking and SSW type can be ascribed to biases in the model's blocking climatology.

\subsection{SSW and the Polar Stratopause}

[39] The average altitude of the polar stratopause during Northern Hemisphere winter in WACCM is about $55 \mathrm{~km}$. Above the stratopause, at around $65 \mathrm{~km}$, there is a maximum in the downwelling branch of the stratospheric Transformed Eulerian Mean (TEM) circulation, $\bar{w}^{*}$ [Andrews et al., 1987]. Figure 9 shows the WACCM climatology of polar cap temperature (defined here as the cosine-weighted average from $70^{\circ}$ to the pole) for simulation refb1.4. The height of the stratopause remains roughly constant in winter until
mid-March, when it descends rapidly to around $48 \mathrm{~km}$ by the end of April, and its temperature increases. This marks the transition to the summer regime. During the summer, the zonal wind (not shown) becomes easterly, and $\bar{w}^{*}$ (shown as superimposed line contours in Figure 9) reverses sign, such that there is upwelling in the polar cap, throughout the stratosphere and mesosphere. In the upper mesosphere, there is a deep temperature minimum (the polar summer mesopause), which coincides approximately with the region where upwelling is strongest. These features show that the polar cap temperature in the mesosphere is dynamically controlled, that is, it is dominated by the effect of adiabatic cooling in summer and warming in winter. Note, by contrast, that the temperature of the summer polar cap stratosphere, below $50 \mathrm{~km}$, follows the annual cycle of radiative heating, being warmest in summer even though $\bar{w}^{*}$ is upward in that season.

[40] The seasonal cycle of polar cap temperature in the mesosphere suggests that this region is highly susceptible to variability in $\bar{w}^{*}$. This variability arises from changes in the driving of the mean meridional circulation by planetary and gravity wave dissipation. A hint that such variability is important is given by the changes in polar cap temperature seen in Figure 9 during late December and January, when the altitude and temperature of the polar cap stratopause change appreciably and their intraseasonal variability increases, even in the climatological mean. In fact, recent observational and modeling studies [Manney et al., 2008a, 2009; Marsh, 2011; Chandran et al., 2011] have shown that major changes in polar cap temperature and $\bar{w}^{*}$ can occur during major SSW. These "elevated stratopause events" begin with a rapid lowering of the altitude of the polar cap stratopause, accompanied by cooling of the upper mesosphere. This is followed by a period when the polar cap stratopause becomes ill defined and then reforms at a considerably higher altitude. Subsequently, this elevated stratopause descends gradually over a period of several weeks.

[41] We identified elevated stratopause events using the procedure described in section 2, and constructed a composite of all such events in simulation refb 1.4 by using as the central date the time $t_{0}$ when a jump in the altitude of the stratopause is detected. Note that our procedure does not attempt to link elevated stratopause events directly to major SSW since, for reasons that are illustrated and discussed next, there is not a very close correspondence between the two types of event. Both elevated stratopause events and major SSW are triggered by disturbances of the stratosphere and mesosphere due to planetary wave-mean flow interactions, as discussed by Chandran et al. [2011]. However, identification of a major SSW is based upon the behavior of the zonal-mean zonal wind in the middle stratosphere at subpolar latitudes $\left(10 \mathrm{hPa}, 60^{\circ}\right.$ latitude), while elevated stratopause events are defined by an abrupt change in the altitude of the polar cap stratopause. While these two measures are, in fact, often causally related, we find that they do not always coincide because they emphasize behavior in different regions of the middle atmosphere.

[42] Figure 10 (top) shows a composite of elevated stratopause events constructed from simulation refb $1.4 ; 18$ events were detected in the 51 winters examined. The composite shows clearly the descent (to about $45 \mathrm{~km}$ ) and warming $(>260 \mathrm{~K})$ of the stratopause associated with strong cooling of 

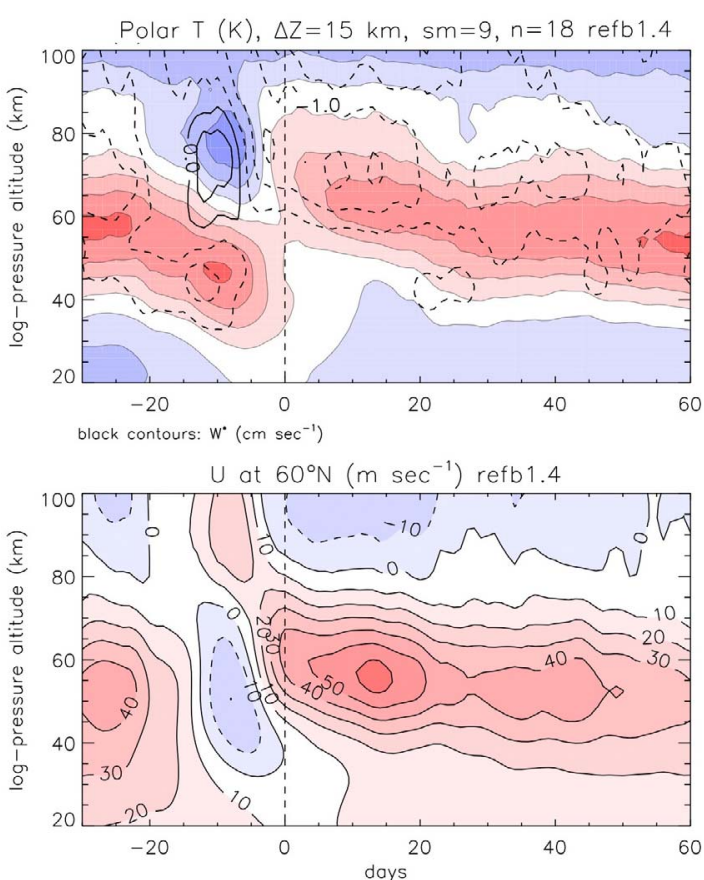

Figure 10. Composites for WACCM simulation refb1.4 of (top) polar cap temperature and TEM vertical velocity, $\bar{w}^{*}$, during "elevated stratopause" events and (bottom) zonalmean zonal wind at $60^{\circ} \mathrm{N}$. The color bar shows temperature contours in $\mathrm{K}$; the TEM vertical velocity is superimposed as heavy contours at $0.5 \mathrm{~cm} \mathrm{~s}^{-1}$ intervals, with negative values denoted by dashed contours. The composite is referenced to the time when the stratopause altitude changes abruptly. See text for details.

the mesosphere $(<200 \mathrm{~K}$ near $80 \mathrm{~km})$. This is accompanied by the formation of a region of strong upwelling in the mesosphere and an enhancement of downwelling at lower altitudes. The correspondence between the direction of $\bar{w}^{*}$ and the behavior of the polar cap temperature indicates that the latter is driven by adiabatic warming and cooling. After the initial period of stratopause descent and mesospheric cooling, the stratopause becomes ill defined and then reforms at altitudes higher than normal $(\sim 75 \mathrm{~km})$, at which time downwelling resumes in the mesosphere. The re-formation of the polar stratopause and its descent to lower altitudes involve changes in gravity wave driving that respond to changes in the stratospheric zonal winds, as discussed by Marsh [2011] and Chandran et al. [2011].

[43] The relationship between the behavior of $\bar{w}^{*}$ over the polar cap and planetary wave-mean flow interaction can be appreciated from Figure 10 (bottom), which shows the zonal wind at $60^{\circ} \mathrm{N}$, composited as in Figure 10 (top), that is, according to the date of the stratopause jump. There is a clear correspondence between the descent of the stratopause before its jump to higher altitude and deceleration of the zonal-mean zonal wind at $60^{\circ} \mathrm{N}$. Indeed, the zonal wind reversal that ensues penetrates to near $10 \mathrm{hPa}(32 \mathrm{~km}$ logpressure altitude), almost enough to qualify as a major SSW, even in this composite view. The zonal wind deceleration is driven by the Eliassen-Palm (EP) flux divergence due to planetary Rossby waves (not shown), the details of which have been addressed by Chandran et al. [2011]. The EP flux divergence responsible for the deceleration of the zonal wind drives a strong mean meridional circulation, whose vertical component, $\bar{w}^{*}$, causes warming below about $60 \mathrm{~km}$ (and cooling above) over the polar cap, as seen in Figure 10 (top). This combination of downwelling below and upwelling above the region of maximum zonal wind deceleration is a well-known feature of the dynamics of SSW [see, e.g., Andrews et al., 1987].

[44] While the composite behavior shows the main characteristics of elevated stratopause events, there are notable differences in timing and evolution among the events that make it necessary to look at individual cases in order to describe completely their behavior. Figure 11 (top) shows an example of a well-developed elevated stratopause event during the winter of 1961-1962 in simulation refb1.4. The features of this event are similar to those seen in the composite of Figure 10, except that the behavior is much more sharply defined, as it lacks the smoothing effect inherent in the compositing procedure. In particular, after dropping to about $45 \mathrm{~km}$, the stratopause rises to above $80 \mathrm{~km}$ and subsequently descends gradually for a period of over two months (late December through mid-March) During this stage, the warming in the middle stratosphere weakens and the stratospheric westerly winds recover [cf. Marsh, 2011; Chandran et al., 2011]. Figure 11 (bottom) shows the behavior of the zonal-mean zonal wind at $60^{\circ} \mathrm{N}$ during the event. The initiation of the elevated stratopause event (29 December 1961) coincides fairly closely with (but is not identical to) the beginning of a major SSW (20 December 1961). The lack of a closer correspondence between the date
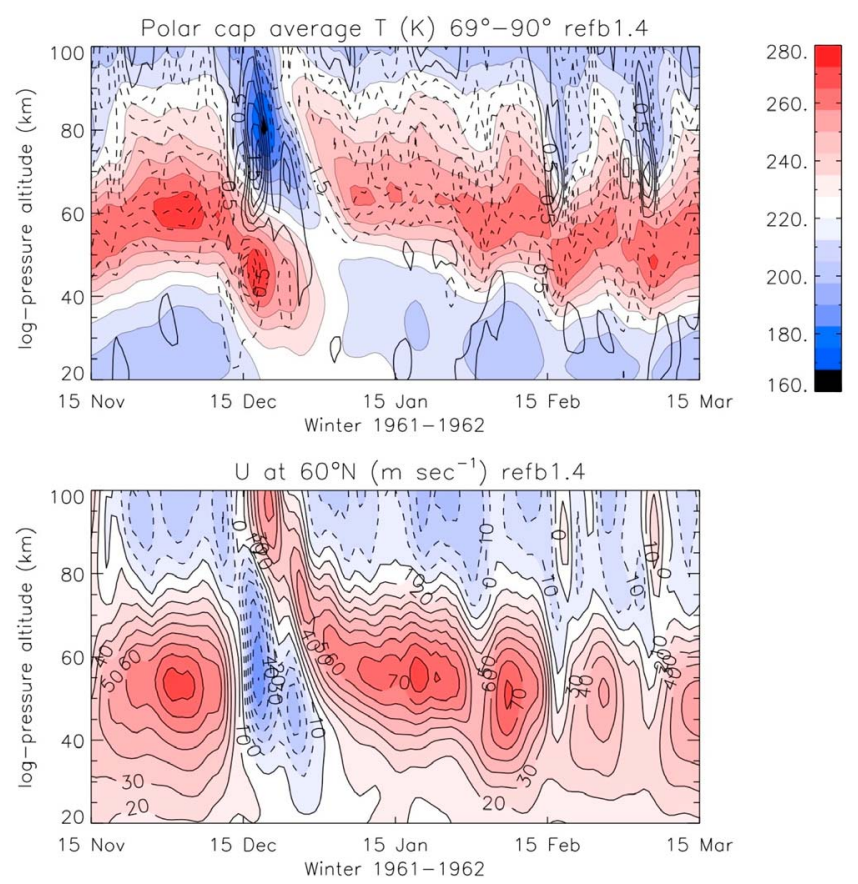

Figure 11. Example of an elevated stratopause event in WACCM. (top) Temporal evolution of polar cap temperature (K; filled contours, color scale on right) and TEM vertical velocity, $\bar{w}^{*}\left(\mathrm{~cm} \mathrm{~s}^{-1}\right.$; line contours at $0.5 \mathrm{~cm} \mathrm{~s}^{-1}$ intervals, with negative values denoted by dashed contours). (bottom) Zonal-mean zonal wind ( $\mathrm{m} \mathrm{s}^{-1} ; 10 \mathrm{~m} \mathrm{~s}^{-1}$ contours, with negative values dashed) at $60^{\circ} \mathrm{N}$. 


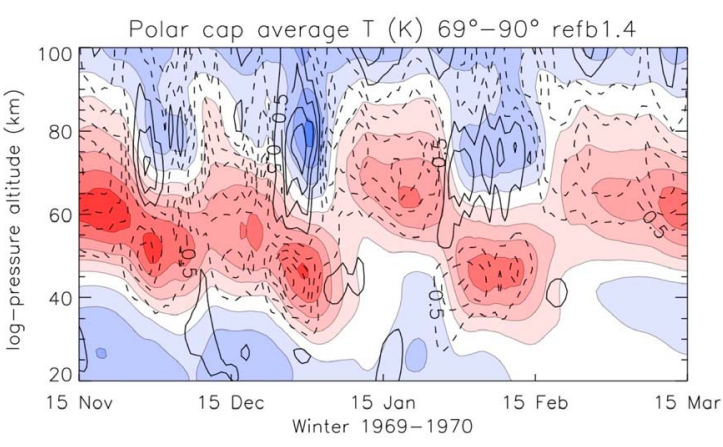

280.

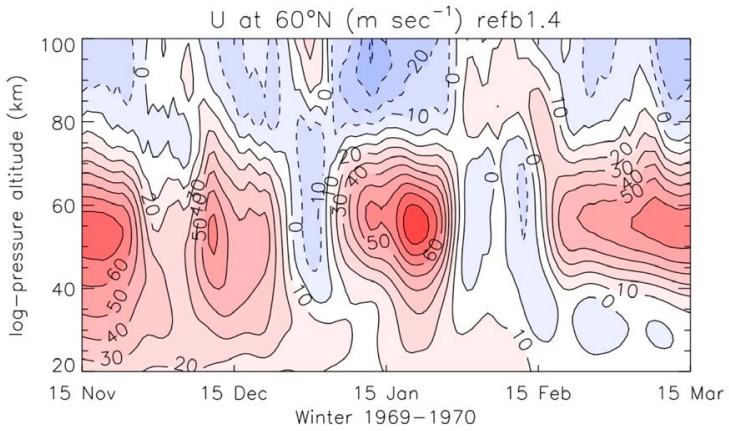

Figure 12. Example of an elevated stratopause event in WACCM associated with two episodes of deceleration of the stratospheric zonal-mean zonal wind. (top and bottom) As in Figure 11. See text for details.

of the elevated stratopause event and the date when the SSW criterion is met is a result of the different definitions used for the two phenomena. Nevertheless, it can be seen clearly from Figure 11 that the polar stratopause temperature responds to the zonal wind deceleration that begins around the middle of December, as it must, since the zonal-mean zonal wind and temperature fields are in approximate geostrophic balance. The SSW and the elevated stratopause are tightly linked, in this example, by the meridional circulation induced through wave-mean flow interaction.

[45] There are other variations in the behavior of elevated stratopause events, which correspond to the details of planetary wave-mean flow interaction. As shown in Figure 10, on average, the stratopause descends to around $45 \mathrm{~km}$ and then reforms at around $70-75 \mathrm{~km}$. The mean difference in the height before and after the stratopause "jump" is thus about 25-30 km. However, the downward displacement of the stratopause can even reach below $30 \mathrm{~km}$, and the altitude where it reforms can be over $85 \mathrm{~km}$. The extreme values compare well with observations made during several recent very strong SSW [e.g., Manney et al., 2008b, 2009]. A large drop of the stratopause altitude during the initial stage of the event is not uniformly related to a subsequent reappearance at very high altitude. The maximum displacement found in WACCM between the lowest altitude reached by the stratopause at the onset an elevated stratopause event and its subsequent reformation at higher altitude was approximately $50 \mathrm{~km}$.

[46] In some events, the polar stratopause altitude drops only slightly, or the height where it reforms is the normal height. The stratopause can even drop and reform more than once during the same event. One such case is illustrated in Figure 12, for the winter of 1969-1970 in simulation refb1.4. Note that the onset of the elevated stratopause event
(7 January 1970) does not correspond closely to the occurrence of a major SSW. There is strong deceleration of the stratospheric zonal wind in January; however, this does not produce a reversal of the wind at $10 \mathrm{hPa}(32 \mathrm{~km})$. Around 10 January 1970, the stratopause reforms near $70 \mathrm{~km}$, but this is followed by another episode of stratopause descent and mesospheric cooling beginning around 1 February 1970. The latter coincides with another period of deceleration of the zonal wind, which finally reverses on 12 February 1970 , the onset of the major SSW in this winter.

[47] The elevated stratopause event illustrated in Figure 12 highlights nicely the relationship between the behavior of the polar cap temperature and that of the stratospheric zonal wind, and demonstrates why there is no uniform relationship between elevated stratopause events and major SSW. Figure 12 shows, further, that the gradual evolution of the polar cap temperatures and TEM circulation seen in Figure 11 (and in the major observed events of 2006 and 2009 discussed by Manney et al. [2008a, 2009]) is by no means universal. Such events require a protracted period of suppressed wave-mean flow interaction after the formation of the elevated stratopause to produce a gradual recovery of the polar cap temperatures (and the associated evolution of the TEM downwelling pattern [see Chandran et al., 2011]). However, Figure 12 shows that the evolution of polar cap temperatures after the onset of an elevated stratopause event can be more complicated if there are repeated episodes of wave-mean flow interaction.

[48] We note, finally, that the WACCM simulations contain frequent instances of elevated stratopause events that occur during winters where no major SSW is diagnosed at all because the zonal-mean zonal wind does not reverse at $60^{\circ}$ and $10 \mathrm{hPa}$. Nonetheless, the evolution of these events resembles closely that of the events wherein a major SSW occurs. In particular, strong deceleration of the stratospheric zonal wind always takes place in connection with the polar cap temperature changes. On the other hand, there are also multiple instances of major SSW where our diagnostic criteria for elevated stratopause events are not met. This happens, for example, when an episode of zonal-wind deceleration affects mainly the middle and lower stratosphere and produces the requisite wind reversal at $10 \mathrm{hPa}$ without, however, affecting strongly the behavior of the polar cap stratopause.

\section{Summary}

[49] This study shows that WACCM (from version 3.5.48 forward) reproduces adequately the main characteristics of major stratospheric sudden warmings. The frequency of SSW, occurrence of displacement and splitting events, amplitude of the SSW, and their dynamical benchmarks are very similar to the results found by Charlton and Polvani [2007] and Charlton et al. [2007] using reanalysis data. The main difference was found for the monthly distribution of SSW frequency, which is biased toward December in WACCM. The internal variability of the model, which determines the difference among the individual runs, is important for the frequency and the interannual variability of SSW. Although WACCM reproduces relatively well the blocking climatology, some of the observed linkages between blocking and SSW were not captured with enough 
realism, particularly as regards the behavior of blocking precursors before SSW of the split vortex type. Our results also suggest that most of the discrepancies in the climatological and dynamical signatures of SSW between WACCM and the reanalyses arise from splitting events.

[50] In previous versions of WACCM, the frequency of SSW was much lower than in the current version. The problem appears to be related to the fact that the horizontal resolution of a climate model such as WACCM is insufficient to account fully for the effects of sharp topographic features. In the present version, a turbulent mountain stress parameterization has been implemented to account for the effect of unresolved orography [Richter et al., 2010]. The parameterization uses a von Karman formulation, where the roughness length is related to the standard deviation of the topography unresolved by the model's relatively coarse horizontal grid. This allows simulation of the momentum exchange between the wind and the nonresolved orography. The wave generation due to mountain ranges such as the Rocky Mountains, which are not well resolved in the model, appears to be important enough to play a major role in increasing the frequency of SSW to realistic values.

[51] We have also examined the occurrence of "elevated stratopause events" in WACCM. In addition to altering the temperature structure of the polar cap, these events are of interest because of the enhanced downward transport that accompanies the descent of the polar stratopause after it reforms at high altitude; this is known to increase the concentrations in the upper stratosphere of minor species, such as $\mathrm{CO}$ and $\mathrm{NO}$, that have sources in the upper mesosphere and lower thermosphere [e.g., Manney et al., 2008b, 2009; Marsh, 2011; Smith et al., 2011].

[52] WACCM is able to reproduce realistically elevated stratopause events, but the simulations also show that there is not a close correspondence between major SSW and elevated stratopause events. Thus, for example, elevated stratopause events can occur when there is strong forcing of the zonal wind and meridional circulation by planetary waves, which nevertheless does not quite produce a wind reversal at $60^{\circ}$ and $10 \mathrm{hPa}$. Similarly, it is possible to have strong wave forcing in the lower stratosphere, which does produce a major SSW according to the conventional criterion, but does not affect appreciably the circulation and temperature structure in the polar cap mesosphere. It is beyond the scope of the present paper to examine in detail the climatology and variability of elevated stratopause events. This topic will be the subject of separate study.

[53] Acknowledgments. We thank A. K. Smith, J. H. Richter, and D. R. Marsh, and three anonymous reviewers for many useful comments, which have helped improved this paper. The National Center for Atmospheric Research (NCAR) is sponsored by the U.S. National Science Foundation. L.T. was partially supported by the Xunta de Galicia while she was a visitor at NCAR. A.C. acknowledges support from the U.S. National Science Foundation under grant ARC 0632387, U.S. International Polar Year program.

\section{References}

Andrews, D. G., J. R. Holton, and C. B. Leovy (1987), Middle Atmosphere Dynamics, Academic, Orlando, Fla.

Barriopedro, D., R. García-Herrera, and R. M. Trigo (2010), Application of blocking diagnosis methods to general circulation models. Part I: A novel detection scheme, Clim. Dyn., 35, 1373-1391, doi:10.1007/s00382-0100767-5.
Castanheira, J. M., and D. Barriopedro (2010), Dynamical connection between tropospheric blockings and stratospheric polar vortex, Geophys. Res. Lett., 37, L13809, doi:10.1029/2010GL043819.

Chandran, A., R. L. Collins, R. R. Garcia, and D. R. Marsh (2011), A case study of an elevated stratopause generated in the Whole Atmosphere Community Climate Model, Geophys. Res. Lett., 38, L08804, doi:10.1029/2010GL046566.

Charlton, A. J., and L. M. Polvani (2007), A new look at stratospheric sudden warmings. Part I: Climatology and modeling benchmarks, J. Clim., 20, 449-469, doi:10.1175/JCLI3996.1.

Charlton, A. J., L. M. Polvani, J. Perlwitz, F. Sassi, E. Manzini, K. Shibata, S. Pawson, J. E. Nielsen, and D. Rind (2007), A new look at stratospheric sudden warmings. Part II: Evaluation of numerical model simulations, J. Clim., 20, 470-488, doi:10.1175/JCLI3994.1.

Eyring, T., G. Shepherd, and D. W. Waugh (Eds.) (2010), Stratospheric processes and their role in climate, Rep. WCRP-132, World Clim. Res. Prog., Geneva, Switzerland. [Available at http://www.sparc-climate.org/ publications/sparc-reports/sparc-report-no5.]

Garcia, R. R., D. R. Marsh, D. E. Kinnison, B. A. Boville, and F. Sassi (2007), Simulation of secular trends in the middle atmosphere, J. Geophys. Res., 112, D09301, doi:10.1029/2006JD007485.

Manney, G. L., et al. (2008a), The evolution of the stratopause during the 2006 major warming: Satellite data and assimilated meteorological analyses, J. Geophys. Res., 113, D11115, doi:10.1029/2007JD009097.

Manney, G. L., et al. (2008b), The high Arctic in extreme winters: Vortex, temperature, and MLS and ACE-FTS trace gas evolution, Atmos. Chem. Phys., 8, 505-522, doi:10.5194/acp-8-505-2008.

Manney, G. L., M. J. Schwartz, K. Krüger, M. L. Santee, S. Pawson, J. N. Lee, W. H. Daffer, R. A. Fuller, and N. J. Livesey (2009), Aura Microwave Limb Sounder observations of dynamics and transport during the record-breaking 2009 Arctic stratospheric major warming, Geophys. Res. Lett., 36, L12815, doi:10.1029/2009GL038586.

Marsh, D. (2011), Chemical-dynamical coupling in the mesosphere and lower thermosphere, in Aeronomy of the Earth's Atmosphere and Ionosphere, IAGA Spec. Sopron Book Ser., vol. 2, 1st ed., edited by M. Abdu, D. Pancheva, and A. Bhattacharyya, pp. 3-17, Int. Assoc. of Geomagn. and Aeron., Dordrecht, Netherlands, doi:10.1007/978-94-007-0326-1_1.

Martius, O., L. M. Polvani, and H. C. Davies (2009), Blocking precursors to stratospheric sudden warming events, Geophys. Res. Lett., 36, L14806, doi:10.1029/2009GL038776.

Richter, J. H., F. Sassi, and R. R. Garcia (2010), Toward a physically based gravity wave source parameterization in a general circulation model, J. Atmos. Sci., 67, 136-156, doi:10.1175/2009JAS3112.1.

Richter, J. H., K. Matthes, N. Calvo, and L. J. Gray (2011), Influence of the quasi-biennial oscillation and El Niño-Southern Oscillation on the frequency of sudden stratospheric warmings, J. Geophys. Res., 116, D20111, doi:10.1029/2011JD015757.

Schwierz, C., M. Croci-Maspoli, and H. C. Davies (2004), Perspicacious indicators of atmospheric blocking, Geophys. Res. Lett., 31, L06125, doi:10.1029/2003GL019341.

Siskind, D. E., S. D. Eckermann, L. Coy, and J. P. McCormack (2007), On recent interannual variability of the Arctic winter mesosphere: Implications for tracer descent, Geophys. Res. Lett., 34, L09806, doi:10.1029/ 2007GL029293.

Smith, A. K., R. R. Garcia, D. R. Marsh, and J. H. Richter (2011), WACCM simulations of the mean circulation and trace species transport in the winter mesosphere, J. Geophys. Res., 116, D20115, doi:10.1029/ 2011JD016083.

Taguchi, M. (2008), Is there a statistical connection between stratospheric sudden warming and tropospheric blocking events?, J. Atmos. Sci., 65, 1442-1454, doi:10.1175/2007JAS2363.1.

Wolter, K., and M. S. Timlin (2011), El Niño/Southern Oscillation behavior since 1871 as diagnosed in an extended multivariate ENSO index (MEI ext), Int. J. Clim., 31, 1074-1087, doi:10.1002/joc.2336.

Woollings, T., A. Charlton-Perez, S. Ineson, A. G. Marshall, and G. Masato (2010), Associations between stratospheric variability and tropospheric blocking, J. Geophys. Res., 115, D06108, doi:10.1029/2009JD012742.

Woollings, T., J. G. Pinto, and J. A. Santos (2011), Dynamical evolution of North Atlantic ridges and poleward jet stream displacements, J. Atmos. Sci., 68, 954-963, doi:10.1175/2011JAS3661.1.

D. Barriopedro, Departamento de Astrofísica y Ciencias de la Atmósfera, Instituto de Geociencias, Universidad Complutense de Madrid, Avenida Complutense s/n, E-28040 Madrid, Spain.

A. Chandran and R. R. Garcia, National Center for Atmospheric Research, Boulder, CO 80307-3000,USA. (rgarcia@ucar.edu)

L. de la Torre, EPhysLab, Facultad de Ciencias, Universidad de Vigo, Ourense E-32004, Spain. 